\title{
Hacia una sociología de la educación universitaria en el Perú
}

\author{
Jaime Rodolfo Ríos Burga \\ Universidad Ricardo Palma \\ jaime.rios@urp.edu.pe
}

La educación universitaria peruana debe estudiar e
investigar las mejores formas de vida y organización
del Perú como sociedad politica global, contribuyendo
a construir una civilización transcultural de vida como
humanos.

RESUMEN

El presente trabajo busca desde la reflexión teórica sociológica educativa ubicar algunas de las nuevas situaciones y problemáticas, modelos y políticas, presentes en la universidad peruana en la presente transformación global. Un análisis y comprensión en sus contextos específicos de reproducción y discursos que nos llevan a constatar que asistimos hoy a la consolidación de una nueva asimetría estructural socioeducativa en el país.

Palabras Clave: Sociología, educación, teoría, globalización, discursos, políticas educativas.

\section{Towards a Sociology in Peru University Education}

\author{
Peruvian university education should study and \\ research the best ways of life and organization of Peru \\ as a global political society, thus contributing to build a \\ transcultural civilization of life as humans.
}

\begin{abstract}
This work seeks to locate some of the new situations and problems, models and policies, from the educational sociological theoretical reflection present in the Peruvian university in the actual global transformation. An analysis and understanding in their specific contexts of propagation and discourses that lead us to verify that today we are witnessing the consolidation of a new socio-educational structural asymmetry in the country.
\end{abstract}

KeYwords: Sociology, education, theory, globalization, discourses, educational policies. 


\section{Introducción}

La sociología de la educación en el Perú es un campo científico específico por fundar y desarrollar. Las herencias teóricas clásicas de Durkheim, Marx y Weber, entre otros pensadores modernos, deben recogerse en la problematización. Como también el aporte sociológico contemporáneo y actual como los de Luhmann, Bourdieu, Morin, Touraine, Freire, entre otros. Vivimos la cuarta revolución industrial científica tecnológica, la generalización del modo de desarrollo infocomunicacional, la transformación del poder global y la creciente transculturalización de las sociedades. Tendencias que marcan hoy nuevos procesos de individuación, sociabilidad, socialización e identidades socioeducativas. Nuevas situaciones de las que tenemos que dar cuenta en sus especificidades y cambio global.

\section{Un ejercicio de sociología educativa}

La educación es un sistema político cultural que integra a los actores de una sociedad en toda su individuación, sociabilidad, socialización e identidades. Procesa acorde con el modelo civilizatorio, societal, político y sociotecnológico y cultural, la producción y la reproducción del sistema social en su concepción educativa, instrucción, visión, empresa, trabajo, cuerpo y cotidianidad. En su función la educación atribuye calificaciones, educa, socializa y crea una identidad a las personas en sociedad (Dubet, 1998). Así, en el tiempo histórico vemos desarrollarse los modelos clásicos roussoniano, positivista, funcionalista, estructuralista, sistémico. Modelos de educación al servicio directo de las demandas del capital y que hoy se generalizan, pero también contradictoriamente se agotan, pues la educación como una función "social" llega a su fin.

La modernidad educativa clásica como sistema se basó en tres principios básicos: la voluntad de liberar al niño de sus particularismos socializándolo para el mundo del trabajo y la empresa; la afirmación del estado-nación; y la socialización ligada a una marcada jerarquía social (Touraine, 1997). Modelo de educación como sociedad nacional que hoy se transforma radicalmente en otro sistema de educación centrado en el individuo "ciudadano" globalizado. El capitalismo corporativo transnacional redefine la utilidad de la educación acorde con sus intereses de acumulación y poder global procesando una nueva visión, estrategias, valores y funciones en una creciente privatización e institucionalización como creciente desigualdad educativa. Dar cuenta de esta nueva dinámica plantea el desarrollo de una sociología de la educación que vaya más allá de la "paideia funcionalista", del marxismo "dogmático", del constructivismo estructural (Bourdieu, 1967) o de la fenomenología sociológica. 
La modernidad clásica construyó todo un sistema educativo asimétrico asignándole una peculiar socialización (Luhmann, 1998). Hoy, el modelo neoliberal lo transforma bajo nuevas formas de diferenciación interna y entorno entre lo local y global planteando nuevos desafíos. Como destaca en un último informe del Banco Mundial su vicepresidente para América Latina y el Caribe Jorge Familiar: "La educación superior es clave para incentivar el crecimiento y reducir la pobreza y la desigualdad. Para asegurar la igualdad de oportunidades, la región debe mejorar la calidad de su educación y brindarles a sus estudiantes una mejor información respecto a programas, incentivos adecuados y opciones de financiamiento, así como generar nexos con el mercado de trabajo. También se debe optimizar la normativa que regula a las instituciones de educación superior para mejorar su rendición de cuentas respecto a los servicios que prestan" (Familiar, 2017).

La universidad se ve inmersa en una creciente interdependencia y competitividad global donde no posee ya ninguna independencia absoluta respecto del sistema global. Funcionan como un dispositivo de sistemas sociales especializados en la transformación de las personas. En el Perú, por ejemplo, constatamos que la globalización produce una crisis de transición donde el viejo sistema educativo superior queda ya desfasado y un nuevo sistema no puede consolidarse por la propia naturaleza dependiente y el papel de los actores educativos en creciente privatización global. Situación que produce en muchos casos de las universidades públicas un permanente conflicto y crisis de reproducción socioeducativa.

Vemos cómo la educación superior pública y privada produce y reproduce nuevos procesos de inclusión y exclusión global diferenciados de cultura organizacional. Las universidades se adecuan cada vez más a las demandas globales. La educación pública, así mismo, se ve cada vez más excluida o subordinada por el sistema corporativo neoliberal. La nueva estructuración crea en una nueva centralización "elitista" de los cuadros docentes y profesionales en función del capital financiero monopólico. Su función externa, invisible y última es contribuir a la reproducción del nuevo sistema de poder del capitalismo financiero global. En esta dinámica, la máscara de independencia (libertad, autonomía, equidad, etc.) no hace más que disimular las funciones sociales que propaga la nueva ideología neoliberal para llevarlas a cabo con mayor eficacia (Bourdieu y Passeron, 1977). Pues el impacto del modelo produce nuevas formas de inclusión, desigualdad social, diferenciación espacial (ciudad/campo), estratificación y movilidad social. Podemos ilustrar esta reproducción en la universidad peruana en el siguiente esquema: 
Perú: MOdelo de ReProduCCIÓN DE UNA UNIVERSIDAD DEPENDIENTE

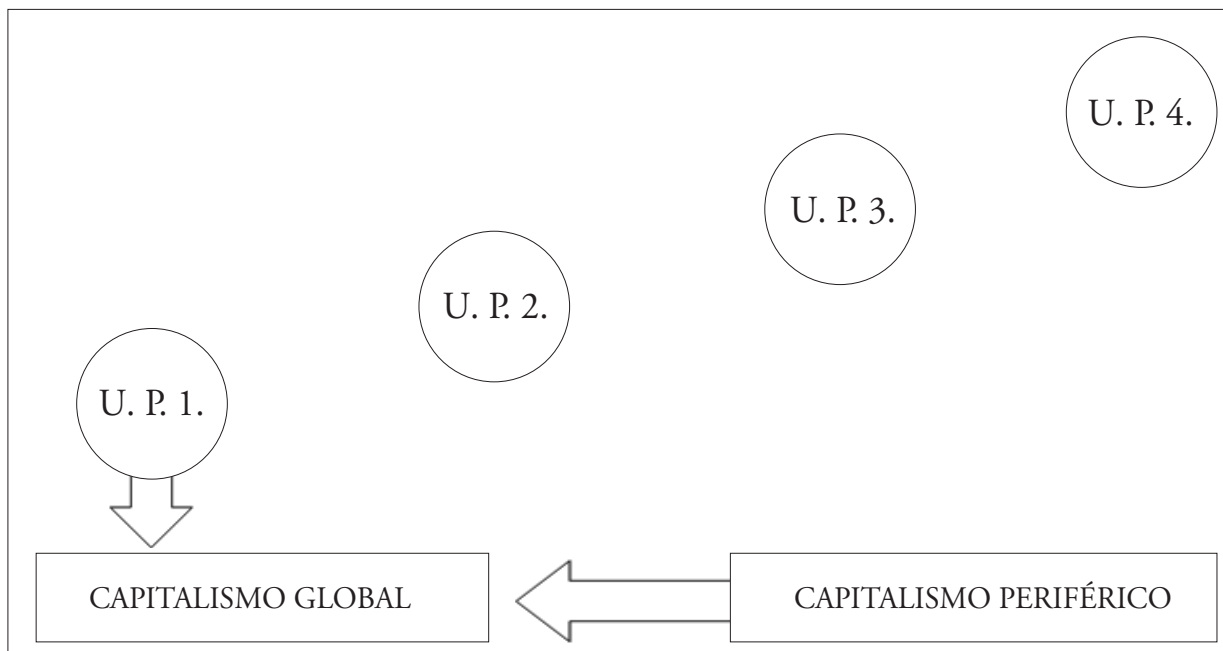

U. P. 4. = Universidad pública

U. P. 3. = Universidad privada baja, media baja y media: UAP, UIGV, USMP

U. P. 2. = Universidad privada media alta: PUCP, USIL, UL, URP

U. P. 1. = Universidad privada globalizada: UP, UPC- Laureate Internacional Universities.

FUENTE: Elaborado por el autor.

\section{Los discursos educativos globales}

Investigar el impacto del capitalismo global en la educación en todos sus niveles es tarea científica de la sociología de la educación. Los discursos educativos van hoy entre modelos de racionalidad instrumental, de reflexión sistémica e histórica. Analicemos los discursos más saltantes para ilustrar las modalidades de su reproducción político cultural educativo global.

\section{El modelo educativo neoliberal}

La consolidación del modelo educativo neoliberal nos lleva a constatar que predominantemente en la región se va perdiendo la idea y la práctica educativa de construir un proyecto educativo nacional global. La educación pública sigue los pasos de la educación privada, se inscribe de manera desigual a la dinámica del cambio global. Vemos como entre la creatividad e imitación se van construyendo nuevas institucionalidades 
universitarias en una competitividad de las "élites" por captar los nuevos mercados educativos (De Azevedo, 1997).

Como destacó Mariátegui para el siglo XX, principalmente la imitación marca el contenido del pensamiento, la acción, la organización y las políticas, en la vida universitaria, reproduciendo formas de colonialidad (Mariátegui, 1973). El capitalismo financiero exige que las universidades resuelvan las demandas crecientes del mercado global y las nuevas expectativas de las nuevas clases medias y las demandas populares de democratización educativa. El proyecto educativo promovido por el Banco Mundial, FMI, OCDE, OMC, etc., promueven una propuesta corporativa sistémica de gobernabilidad educativa.

Sus políticas fomentan la mayor diferenciación de las instituciones públicas y privadas, la creación de incentivos para que las instituciones públicas diversifiquen las fuentes de financiamiento, la redefinición de la función del gobierno en la enseñanza superior, la adopción de políticas que estén destinadas a otorgar prioridad a los objetivos de calidad y equidad, la privatización de la educación, la supresión de la gratuidad implementando el cobro de matrícula, la creación de instituciones no universitarias a nivel terciario, y el quitar la prioridad a la investigación en las universidades públicas (BM, 1995; Yarzábal, 1999; Arnold, 2000; Brunner, 2000; Rodríguez, 2000).

En su impacto produce una diferenciación socioeducativa entre lo privado y lo público. Una constante estructural del sistema educativo neoliberal. Excluye a todo aquel que se oponga a su modelo altamente centralizado y de control sistémico (Bauman, 1999; Chomsky y Dieterich, 1997; Castells, 2002). Por ejemplo, si bien en el discurso destaca reconocer la importancia de la educación superior para el desarrollo económico y social, su mayor diversificación, incluir el desarrollo de instituciones privadas, incentivos a las instituciones públicas, mejora del rendimiento académico e institucional, optimización de la calidad y al fomento de la equidad, calidad y eficiencia, calidad de la enseñanza y la investigación, demandas del mercado laboral y a las cambiantes demandas económicas, incrementar la equidad y desarrollo institucional (BM, 1995).

La aplicación del modelo como muestra la experiencia peruana y chilena nos presenta resultados diferenciados de inclusión y exclusión social, produciendo en perspectiva cada vez más asimetrías (Ferreyra, Avitabile, Botero, Álvarez, Haimovich y Urzúa, 2017) y agotamiento, como muestra la experiencia chilena. Jorge Rojas, al referirse a esta experiencia, destaca que la política de macrotransformación neoliberal educativa en Chile destruye los entramados de la vida individual y colectiva en la sociedad, reproduciendo cada vez más un individuo inhumano, agresivo, depresivo, física y psíquicamente enfermo y en rupturas con el otro y sus entornos. La cultura del mercado por el mercado no crea una cultura de vida sino una cultura individualista cada vez más competitiva. 
El modelo expresa una práctica política basada en el control y dominio total de los actores socioeducativos donde el Estado-nación se diluye y subordina al capitalismo financiero como simples "clientes" o "consumidores". Un modelo que se construye hacia afuera, destruyendo hacia adentro toda forma de organización social e identidades que no sea la de los intereses de su racionalidad en el mercado especulativo global. Un mercado de carreras universitarias que tiene para existir adecuarse a los mercados diferenciados, descuidando una educación de calidad para todos (Rojas, 2012). Un último informe del Banco Mundial muestra que "el progreso no necesariamente ha sido equitativo" porque, en primer lugar, varios de los países de ingreso bajo aún están lejos de cumplir los objetivos básicos establecidos como objetivos de desarrollo del milenio (ODM): educación primaria universal según las mediciones de las tasas de matriculación y de terminación de la educación primaria, e igualdad de género en las matrículas en las escuelas primarias y secundarias para el año 2015. En segundo lugar, en casi todos los países, aunque en distintos grados, existen grupos poblacionales cuyo progreso educativo se ha retrasado debido a que se encuentran en desventaja por cuestiones relacionadas con sus ingresos, género o pertenencia etnolingüística. Dejar a esos grupos desfavorecidos y carentes de recursos al margen del sistema educativo terminará por menoscabar la capacidad del país de mantener el progreso educativo y lograr el desarrollo socioeconómico" (BM, 2017).

\section{El modelo liberal de la Unesco y la Cepal}

La Organización de la Naciones Unidas para la Educación, la Ciencia y la Cultura (Unesco) prioriza la educación para alcanzar el desarrollo humano sustentable. Las instituciones educativas deben elaborar en consulta y coordinación con todos los sectores «una nueva visión ", objetivos, tareas y funcionamiento, de cara a los desafíos del siglo XXI. Su diagnóstico plantea ante la diversificación garantizar la calidad de las instituciones y programas, reexaminar las relaciones entre la educación superior y la sociedad civil, el universo de trabajo y el sector productivo. El sistema de educación superior debe ser suficientemente flexible para hacerle frente a los retos de un mercado de trabajo rápidamente cambiante, resaltar los valores éticos y morales en la sociedad, las relaciones constructivas entre estado y universidad, renovar los métodos de enseńanza-aprendizaje y destacar el lugar de la docencia, buscando el conocimiento y el sentido de responsabilidad para poner su formación al servicio del desarrollo social (Unesco, 1995).

Un modelo que al reunir la diversificación flexibiliza la organización de la universidad en sus estructuras académicas y métodos de enseńanza. Evoluciona hacia la integración de un sistema nacional de educación superior universitaria permanente para la formación, la actualización y el reentrenamiento. La innovación en permanente 
transformación curricular y métodos de enseńanza-aprendizaje. Y la demanda de mercado para lo cual se debe dar relevancia y competitividad a los graduados. Un sistema de educación lo suficientemente flexible para hacer frente a un mercado de trabajo rápidamente cambiante.

Es decir, ya no se puede aplicar más la ecuación: título = trabajo. La educación superior debe producir egresados que no solo puedan ser buscadores de trabajo, sino también empresarios de éxito y creadores de empleo. Un nuevo paradigma donde la relación Universidad-Sociedad-Empresa (Valdés, 1996) superen las brechas entre los sistemas educativos y las necesidades de las empresas planteando nuevas formas de aprendizaje y educación. Para ello se hace clave replantear toda la visión educativa anterior enseńada a pescar, cómo generar nuevos conocimientos, producir pensamientos nuevos, es decir, se ha enseńado todo tipo de pensamientos, pero no el proceso y la mecánica para llegar a ellos. Se ha enseñado a creer ciegamente en un paradigma, pero no se ha enseñado a romper con él y a crear nuevos.

En esta perspectiva, Tünnermann (1996) propone construir una visión prospectiva que lleve a la universidad a la elaboración de los proyectos futuros de sociedad, inspirados en la solidaridad, la equidad y el respeto del ambiente. Llevar a la práctica las nuevas necesidades como las planteadas por la Declaración Mundial sobre Educación Superior en el Siglo XXI (1998): construir una visión, misión y acción educar, formar y realizar investigaciones. Su función ética, autonomía, responsabilidad y prospectiva. Igualdad de acceso. Fortalecimiento de la participación y promoción del acceso de las Mujeres. Promoción del saber mediante la investigación en los ámbitos de la ciencia, el arte y las humanidades y la difusión de sus resultados. Orientación a largo plazo fundada en la pertinencia. Reforzar la cooperación con el mundo del trabajo y el análisis y la previsión de las necesidades humanas. La diversificación como medio de reforzar la igualdad de oportunidades. Métodos educativos innovadores: pensamiento crítico y creatividad. El personal y los estudiantes, principales protagonistas de la educación superior. Evaluación de la calidad. El potencial y los desafíos de la tecnología. Reforzar la gestión y el financiamiento de la educación superior. La financiación de la educación superior como servicio público. Poner en común los conocimientos teóricos y prácticos entre los países y continentes. El retorno de la "fuga de cerebros». Las asociaciones y alianzas.

Modelo que cobra cada vez más importancia a partir de la reunión del «Grupo Asesor en Educación Superior de Unesco», quien identificaba la integración entre docencia e investigación, tomar medidas para asegurar la democratización y promover la calidad de la educación superior. Asimismo, la diversificación de los sistemas de educación superior y su vinculación con el sector productivo, afrontando el impacto de la globalización en los planes y programas de estudio de las instituciones de educación 
superior. Para ello, las universidades deben establecer nuevos lazos con la sociedad y convertirse en lugares para aprender durante toda la vida (Unesco, 1998).

Una evaluación de impacto del modelo en la región muestra una nueva dinámica socioeducativa diferenciada y asimétrica. Destaca uno de sus estudios: "Sin embargo, dada la realidad latinoamericana, se debe tener conciencia del pero que tienen las desigualdades y exclusiones al interior de estos países. A menudo hay una disociación entre los objetivos universales que proponen las políticas sociales (educación, salud, vivienda) y los territorios en los cuales se aplican, en especial con respecto a las ciudades. El problema está en que las ciudades nunca han sido territorios homogéneos, están compuesta por diferentes elementos: áreas centrales, periferias pobres, enclaves ricos y afluentes, zonas industriales y de servicios, concentraciones de sectores vulnerables, etc. Estas divisiones internas se han intensificado en los últimos años con la concepción neoliberal de la ciudad como mercado, en la cual el espacio urbano es una mercancía, y las diferencias internas se han intensificado" (Unesco, 2014).

La propuesta en la teoría y la práctica contiene un discurso humanista universal que se inspira en la tradición de la modernidad clásica del individuo ciudadano, del estadonación y la sociedad civil como paradigma social. Encontrando su mayor desarrollo teórico sistémico en el planteamiento de Edgar Morin: "La educación debe conducir a una antropo-ética considerado el carácter ternario de la condición humana cual es el de ser a la vez individuo sociedad especie. En este sentido, la ética individuo especie necesita un control mutuo de la sociedad por el individuo y del individuo por la sociedad, es decir la democracia; la ética individuo especie convoca la ciudadanía terrestre en el siglo XXI" (Morin, 2001).

El modelo no procesa una crítica integral al carácter del capitalismo global e impacto de la política educativa neoliberal. Su visión y enfoque se sustenta también en el individualismo racionalista, no saca a luz las verdaderas causas y mecanismos de la crisis educativa contemporánea y actual: la creciente desigualdad educativa global producto de la aplicación del modelo y política neoliberal. Pero sin duda contribuye a tomar conciencia del dilema educativo global: ¿en qué tipo de civilización queremos vivir: en una sociedad en creciente desigualdad, violencia y guerras o en una civilización universal del buen vivir o bienestar y paz?

En nuestra experiencia vemos cómo el capitalismo global transforma totalmente el imaginario socioeducativo del estado-nación continental. Vemos construirse nuevos modelos de universidad en el marco sistémico del capitalismo mundial. Un análisis de los discursos de sus actores típicos nos ilustrará los nuevos modelos y políticas presentes.

Un primer modelo global lo plantea Trahtemberg. "No basta parchar el pasado", se hace necesario observar la ley universitaria 30220. El andamiaje estratégico propuesto no se ajusta a la dinámica corporativa educativa global, por tanto, al desarrollo de las capacidades y las competencias que exige la dinámica corporativa. En estas condiciones, 
la elaboración de la tesis, la elección universal, la construcción de los currículos supervisados por la Sunedu, la falta de una visión de las nuevas carreras, el creditaje, el divorcio entre la educación técnica y la universitaria, la no consideración de la educación virtual, el carácter de la ley y el presupuesto bajo la nueva ley garantizan una universidad de calidad, apostando por construir una alianza con los centros corporativos (Trahtemberg, 2014). En su perspectiva, a la política no le interesa construir un sistema educativo nacional global, solo le cabe una incorporación subordinada al sistema capitalista corporativo global.

Un segundo modelo corresponde a la política educativa que se implementa desde el gobierno de Fujimori a la actualidad. En su continuidad viene sustentado por el exministro de Educación Jaime Saavedra. Plantea una política de estado centralizada a un sistema de control que va más allá de la propia autonomía universitaria. El licenciamiento va unido a tres ejes estratégicos: un sistema de información, políticas de fomento y un sistema de acreditación. El sistema de información brindaría bajo la supervisión de la Sunedu todas las características de las universidades en sus respectivos mercados. Las políticas de fomento como subsidios en becas y otros mecanismos crediticios para los alumnos. Las políticas de acreditación a partir de políticas de subsidios a la investigación y mejora de los planes pedagógicos unida a la acreditación internacional, reformulando la ley marco del Sistema Nacional de Acreditación de la calidad. La política económica integra la política educativa en una homogeneidad de estándares medibles (Saavedra, 2014). A diferencia del primer modelo, su fortaleza reside en construir y canalizar como política pública el mercado educativo nacional para la globalización acorde con las políticas del Banco Mundial. Presenta dos debilidades estructurales: no responde a una política nacional global que articula objetivos y metas de los peruanos; y su propia lógica centralista burocrática no refleja la compleja dinámica de intereses existentes en la educación universitaria nacional.

Un tercer modelo global es el planteado por ex rector de la PUCP Salomón Lerner Febres. La universidad tiene el desafío de su internacionalización mediante redes universitarias como elemento estratégico de su desarrollo. Proceso que pone en cuestión la identidad de las instituciones, pero a su vez abre el camino para su diversidad sin renunciar a la independencia. De ahí la tarea de formar ciudadanos globales. Un modelo diferente al modelo universidad-empresa que mira solo lo económico, perdiendo los fundamentos científicos, humanísticos y éticos acorde con las demandas regionales sin caer en modelos únicos hegemónicos que imponen sus lógicas de poder, cultura y saber. Una universidad que promueva la cooperación y el respeto por un mercado verdaderamente libre para todos, donde la calidad educativa constituya un elemento decisivo de un proyecto de desarrollo nacional a partir del papel y función promotora y reguladora del estado con presupuestos adecuados (Lerner, 2014). Su fortaleza ideal se ubica en la tradición humanista cristiana de universalización de la educación, su debilidad en no 
tomar en cuenta que en la etapa de financiarización global se da una profunda diferenciación asimétrica de los actores socioeducativos, producto de la propia racionalidad socioeconómica y sociocultural en la competitividad sistémica corporativa global.

Los tres modelos operan ya en el Perú, pero en creciente privatización e internacionalización. La universidad pública queda rezagada por la incapacidad de sus propios actores internos de adecuarse a estos cambios o revolucionar democráticamente el modelo de universidad hegemónica. Si los dos primeros modelos como patrón sistémico organizacional universitario prevalecen en el tiempo, se consolidará un sistema universitario predominantemente privado dependiente. La universidad pública sufrirá bajo modelos no democráticos procesos de incorporación vertical, fragmentada y excluyente en la dinámica global del cambio. Como hemos señalado, la experiencia chilena es ilustrativa al respecto.

\section{Dos proyectos en debate global}

El modelo neoliberal apuesta por una educación en la desigualdad. Como heredera del positivismo, se consolida como política, pero contradictoriamente se agota en las propias irracionalidades del sistema. Por otra parte, vemos gestarse desde los actores y movimientos sociales democráticos universitarios una nueva visión que plantea una educación superior como un derecho universal y paradigma transcultural de vida desde sus actores (Ríos, 1998). Dos modelos civilizatorios que luchan por dar sentido de vida a los humanos. El modelo neoliberal sistémico con su filosofía pragmatista del mercado, poder y guerra (Petras, 2002), organizando la vida social bajo una racionalidad estratégica de dominación (Hirschman, 1991). Y el modelo educativo transcultural que, bajo una cultura civilizatoria de vida (Morin, 2018; Boron, 1997), organiza el conocimiento y la sociedad como política de vida (De Sousa, 2007) en condiciones de la nueva revolución científica tecnológica (Castells, 2002).

El capitalismo global ubica diferentes teorías. La teoría del capital humano, la teoría de calificación humana, las teorías estructurales, etc. Todas ellas reducen la vida social a la simple reproducción individualista de lo humano como capital en el mercado. Pero, en la hegemonía de la visión y política socioeducativa instrumental, reflexiva sistémica o individualista sale a luz la creciente crisis del ser, la sociabilidad y la socialización e identidades. Vemos desarrollarse una nueva actitud y aptitud formativa y profesional que vaya más allá de la propia racionalidad moderna. Los actores educativos, aparte de su eficacia frente al mercado, anhelan el desarrollo de su personalidad, su vida y sus proyectos personales y colectivos en sus entornos y el cambio global (Touraine, 1997).

La herencia del humanismo se enriquece desde una filosofía transcultural de vida. Transforma el humanismo liberal abstracto en una práctica de una política educativa 
de vida donde ya no basta formar y fortalecer la libertad del sujeto personal, el reconocimiento de la diversidad y del otro, corregir la desigualdad de las situaciones y oportunidades, sino resolver el carácter instrumental mismo de la construcción social e individual de la personalidad en el capitalismo corporativo. Una superación como especie y cultura, como seres vivos y humanos. Las demandas de democratización de las sociedades plantean cada vez más la construcción de nuevos paradigmas ante la soledad del individualismo socioeducativo.

El modelo neoliberal no resuelve los problemas de la vida, su impacto es devastador; el caso del empleo, verbigracia, es un buen ejemplo por su efecto concentrador y mínimo. La igualdad de oportunidades no se da. Las diferencias estructurales acentúan el poder de unos pocos, la autonomía relativa de los actores frente al sistema y sus entornos produce un desfase estructural tanto de la educación pública como de parte importante de la educación privada. El desempleo de los egresados de las universidades y la diferenciación de las universidades unidos a la concentración científico tecnológico se acentúan y marcan la variabilidad de la calidad de la educación en el conjunto social.

Es así que vemos surgir proyectos de construcción de políticas nacionales globales como parte proyectos civilizatorios globales. La interdependencia funciona aquí como la capacidad de los actores educativos de responder a las demandas que plantean el cambio civilizatorio y el capitalismo global sin perder de vista las especificidades étnicas, nacionales como seres vivos. El problema educativo en su esencia sigue planteándose como un problema económico social, político, ético y cultural. Las universidades públicas y privadas se integran como organizaciones inteligentes en alianzas que buscan resolver nuestros problemas concretos y de dependencia estructural de los centros de poder global, afirmando los derechos y deberes, sustentabilidad y sostenibilidad global (Villaseñor, 2003).

Un sistema educativo abierto al mundo que se propone formar y provocar los mejores hábitus presentes, ejerciendo una acción estratégica que nos lleva a realizar todas nuestras potencialidades como personas en sociedad y viceversa. Una visión única y diversa de cada una de nuestras diversidades regionales al servicio directo de nuestros pueblos y naciones en integración global.

En la unidad de nuestras diferencias construimos este modelo teórico-práctico desde lo profundo de nuestras experiencias socioculturales con decisión y voluntad política. La educación se convierte en un medio y fin de vida. La imaginación y la creatividad nos llevan con coherencia y claridad a la verdad, aprender a aprender y a ser personas en sociedad incorporando los siete saberes que plantea Morin (2001) para afrontar los desafíos globales: superar las cegueras del conocimiento: el error y la ilusión; promover un conocimiento capaz de abordar los problemas globales, enseñar la condición humana, la identidad terrenal, enfrentar las incertidumbres, enseñar la comprensión y la ética 
del género humano. Tarea civilizatoria que en nuestra realidad debe siempre ir unida a la descolonización de nuestra situación de dependencia.

Un proyecto nacional global transcultural que con sus investigaciones da cuenta del impacto de las variables estratégicas de la nueva racionalidad del cambio civilizatorio, promoviendo un proyecto compartido desde los actores en definidos planes, programas, políticas y metas: el desarrollo de una estrategia de desarrollo de vida y humano sustentable; su internacionalización mediante planes, programas y actividades en una adaptación dinámica y cambiante en todos sus niveles; y globalizar nuestra integración como nación única y diversa en la sociedad mundial transcultural.

Investigar las mejores formas de organización como sociedad, el conocimiento del papel de las empresas transnacionales y su lógica sistémica de poder, el papel de los estados nacionales dominantes o bloques hegemónicos, y los efectos de la lógica del capitalismo global en su conjunto. Todo como estrategias y políticas concretas que resuelvan los profundos problemas del capitalismo contemporáneo, de la sociedad peruana, pueblos y estados en integración democrática global.

Así mismo, la investigación contribuye a organizarnos como sociedad nacional en integración abierta al cambio global, revolucionando las condiciones económicas, sociales, políticas, científicas y culturales. Propagar los conocimientos avanzados como tecnología de vida vinculados a la solución de los problemas esenciales del Perú-Mundo acorde al cambio global (Ortiz, 1996). Cada nivel educativo debe construir un sistema único e integrado, flexible y dinámico. Partimos de la idea que los problemas y sus soluciones son globales, los construimos todos los días, orientándonos por una actitud democrática sustantiva que niega toda práctica autoritaria, clientelista o de grupo de interés. A la lógica centrista e individualista del capitalismo neoliberal le oponemos una racionalidad social, científica, ética y moral solidaria global estrechamente vinculada a la vida de nuestros pueblos y la humanidad como un todo.

\section{Los ejes estratégicos del cambio}

Los ejes estratégicos de esta transformación educativa se enmarcan en los procesos de democratización sustantiva de la sociedad mundial como liberalización positiva de todas las potencialidades de las personas, los pueblos, los estados nacionales, los grupos en integración, a partir de la unidad y diversidad cultural civilizatoria mundial. Una realización del ser y el saber que a la vez que descoloniza cotidianamente el poder (Quijano, 2014, 2000) integra la vida personal y social a una sociedad transcultural mundial de vida.

Hoy que los espacios y tiempos personales se transforman, nos corresponde ser partícipes de la construcción civilizatoria de una nueva concepción del desarrollo de la 
vida. Elevar la calidad de vida a partir de mercados sociales integrados, un desarrollo intercultural dinámico y la solución de la crisis del sistema histórico moderno. Un sistema social flexible y abierto en redes de investigación científica, tecnológica y social, afirmando una "racionalidad sustantiva" de vida. "Las universidades jugarán el papel de ser un locus importante (por supuesto no el único) de análisis de las realidades de nuestro sistema-mundo. Son estos análisis los que pueden hacer posible navegar en la caótica transición hacia un nuevo orden mundial, que esperamos sea mejor. Al momento, los disturbios al interior parecen no ser más fáciles de resolver que los disturbios en la economía-mundo" (Wallerstein, 2012).

Unas estrategias que no se reducen a un modelo para una "élite", donde la actividad intelectual es solo "propiedad" de una determinada categoría de "especialistas", sino de una sociedad red política organizada como humanos peruanos universales. Visión que afirma una sociedad de vida y de conocimiento como atributo de la cultura científica de los pueblos. Una cultura científica que resuelve en integración sus problemas y supera toda forma de explotación, dominación, desigualdad, pobreza, destrucción ecológica. Un modelo cualitativamente diferente al modelo del pensamiento único neoliberal: individualismo — público + privado, poder dinero = mercancía, limitaciones éticasparticipación política, sustitución de la cultura por la moda, consumismo (industria cultural), relativismo cultural y fin de la historia e ideologías.

Como peruanos, nos corresponde organizar una sociedad nacional global con un proyecto de educación permanente para todos (Unesco, 1998). La globalización de la vida social no niega nuestra afirmación como comunidad educativa nacional; por el contrario, la enriquece. Nuestras universidades contribuyen a orientar con sus investigaciones las políticas del cambio civilizatorio, a promover nuevas carreras e investigaciones multidisciplinarias que resuelvan los profundos problemas de nuestros pueblos y la humanidad toda. La universidad cumple aquí un nuevo papel de liderazgo transformador con una visión global compartida al servicio de la vida en todas sus formas. La mayor debilidad de la universidad en el Perú es no haber sabido integrarse a su sociedad en una unidad de intereses. El elitismo de casta o grupo ha sido uno de los factores de extrañamiento que junto con la dependencia intelectual foránea está renunciando al ideal vallejiano de que "toda voz genial viene del pueblo y va hacia él” (Vallejo, 1983).

La educación universitaria no se abstrae en un intelectualismo y burocratismo infecundo, afronta los problemas esenciales del país a la búsqueda soluciones a sus problemas esenciales, gestando un bloque intelectual y moral de peruanidad como parte de la sociedad democrática global. Teoría y práctica científica que dialogan fecundamente entre sí y con las otras experiencias educativas del mundo, superando los deseos y anhelos que no van paralelos con una distribución equitativa de los bienes (Abugattás, 1992). Un modelo de liderazgo colectivo transformador como nación transcultural. 


\section{Hacia un programa de investigación}

La organización de la investigación nos conduce a superar la dependencia. El "desarrollo" no nos vendrá de afuera. Políticamente es el modo de transformación de una sociedad a partir de su movilización económica, política, cultural y acción voluntaria consciente de sus actores políticos (Touraine, 1998), con definidas políticas como estado en sociedad. El mito del desarrollo en el siglo XX ha servido para hacer de los países ricos más ricos y de los pobres más pobres, en un verdadero darwinismo internacional (De Rivero, 1998).

cializada, acorde con la solución de nuestros problemas concretos y del sistemamundo. Desarrollar áreas estratégicas como la gobernabilidad nacional global, el nuevo mundo del trabajo, la organización del conocimiento, la tecnología y la sociedad, el medio ambiente, la ciencia y tecnología punta, el control del territorio y sus recursos, la regionalización y descentralización, los mass-media, la educación y el arte. Una visión que promueva el siguiente programa general de investigación:

\section{Hacia un esquema integrado de investigación}

I. La transición hacia una nueva sociedad y universidad

- Visión

- Misión

- Objetivos

- Ejes estratégicos

II. Los obstáculos de la reforma

- Estudios

- Financiación

- Personal

- Investigación

III. El conocimiento, innovación y aprendizaje

- Proceso histórico cultural

- Factores estructurales

- Procesos de innovación

- Nueva cultura científica técnica

IV. El posgrado y entorno societal

- Formación para la vida

- Las necesidades colectivas 
- Acceso creciente

- Política universitaria global

V. La difusión del conocimiento

- Nuevas necesidades

- Diversificación y unidad

- Sistemas de enseñanza

- Titulación

- Sistema de apoyo a la enseñanza

VI. La generación y aplicación del conocimiento

- Nuevas tendencias de la investigación

- Política de ciencia y tecnología

- Sistema público de I + D + I + A

- (Léase como investigación, desarrollo, innovación, aplicación.

- Sistema privado de I + D + I + A

VII.Las líneas de acción

- Problemas esenciales

- Convergencia del sistema peruano de ciencia y tecnología con los bloques en integración mundial.

- Potenciación de la política universidad, sociedad y empresa.

- Fomento público de la investigación básica.

- Política de transferencia y rescate científico tecnológico.

- Fomento de I + D + I + A

- Formación de investigadores en el doctorado acorde con la transformación nacional global

VIII.La financiación

- Pública nacional

- Privada nacional

- Internacional

El posgrado corona aquí el sistema de conocimiento universitario, canalizando toda la creatividad e inventiva en un creciente y elevado nivel de intercambio real y virtual con el mundo. La ciencia libera la teoría en sociedad, superando el esquema clásico moderno (Beck, 1998), en una verdadera revolución socioeducativa y reforma radical de la enseñanza. Es decir, se integra a la democratización real de la sociedad desde una nueva 
epistemología y metodología integrada donde el mercado y las disciplinas realizan un trabajo en equipo transdisciplinario, multidisciplinario e interdisciplinario.

El sistema nacional del posgrado conforma "el consejo peruano del futuro", que presenta propuestas concretas y simuladas de soluciones en todas sus áreas estratégicas. Enriquece el plan y el programa nacional de ciencia y tecnología acorde con los planes y programas de los espacios regionales, promueve el desarrollo local y regional a partir de la creación de verdaderos complejos productivos, industriales, científicos y tecnológicos integrados al mundo y al servicio de la vida social de cada una de nuestras regiones. En esta perspectiva, el sistema universitario peruano no se contenta con ser una sucursal y/o dependencia de tal o cual fundación foránea, de un estado nación potencia o un bloque de integración hegemónico que buscan crear un nuevo sistema de colonización, sino formar profesionales que eleven la calidad de vida de cada una de las regiones del país, en diálogo y cooperación con las otras sociedades y universidades del mundo.

La tecnológica educativa en sus diferentes medios se pone al servicio del estudio, la investigación y la construcción institucional. Una formación integral que recoge lo mejor de la cultura, ciencia y técnica universal. El pregrado y posgrado se integran con la previsión de la dirección y el ritmo del cambio global (Picardo, 2000). Una política de investigación directamente vinculada al servicio de nuestra organización institucional como sociedad del conocimiento, afirmando democráticamente el desarrollo científicotecnológico, los recursos humanos y gerencia nacional global. Una educación integral que asegure cada vez una mejor calidad de vida como especie y humanos. En otras palabras, con Galeano nos resistimos a hacer que "El Sur aprenda geografía en mapamundis que lo reducen a la mitad de su tamaño real. Los mapamundis del futuro ¿lo borrarán del todo? Hasta ahora América Latina era la tierra del futuro. Cobarde consuelo; pero algo era. Ahora nos dicen que el futuro es el presente". Corresponde a la sociología peruana ser parte fundacional de esta nueva sociología transcultural como ciencia social universal, dando cuenta de los fenómenos socioeducativos de la sociedad global en toda la unidad de su diversidad civilizatoria universal.

Un modelo educativo y político peruano universal que recoge lo positivo de las experiencias globales. Vemos surgir desde los propios actores universitarios un encuentro entre lo público y privado, social, nacional, global. Movimientos sociales universitarios nacionales como parte de un movimiento social mundial por la vida. Movimiento que plantea una profunda reforma democrática e institucional de la vida universitaria, planteando el desafío de contribuir a resolver los problemas estructurales globales y de nuestros países integrándose en mercados y comunidades solidarios. Una nueva agenda y gobernabilidad universitaria que respondan a las nuevas situaciones y problemáticas de la vida social. Los propios actores se autoevalúan entre pares e integran también a una universidad extendida virtual en alianzas entre universidades-comunidades-empresas sociales globales. 
En síntesis, creamos un sistema universitario nacional global que se autoproduce como organización inteligente de vida como autoorganización, autorregulación y cultura organizacional (Arnold, 2014), en intercambio de ecosaberes en sistemas abiertos integrados al cambio global. Un modelo multiparadigmático que coordina con entes reguladores globales bajo una prospectiva de una política de vida. El sistema infocomunicacional, las políticas de licenciamiento, las maestrías, los doctorados, el fomento y la acreditación responden a las profundadas necesidades de los mercados sociales en globalización. Un modelo educativo diverso que vincula la economía a la vida social sostenible, revolucionando la calidad de vida individual y colectiva de manera solidaria (Bialakowsky, 2014). Al igual que la generación de la reforma universitaria que celebramos, buscamos también hacer realidad la promesa de la vida peruana (Basadre, 1975), peruanizando al Perú (Mariátegui, 1973) como parte de una civilización transcultural de vida, uniendo lo que la modernidad separó: lo verdadero, lo bueno y lo bello.

\section{Referencias}

Abugattás, J. (1996). Elementos para un debate sobre la universidad. Alma Mater. No 11. Lima: UNMSM.

Abugattás, J. (1992). Marco conceptual de la ciencia y la tecnología. Alma Mater. Lima: UNMSM.

Adell, J. (2002). Tendencias en educación en la sociedad de las tecnologías de información. Revista Electrónica de Tecnología Educativa. No 7 . Recuperado de www.uib. es/depart/gte/revelec $7 . h t m l$

Arnold, M. (2014). Imágenes de la complejidad: la organización de las organizaciones: En Arnold, M. Cadenas, H. y Urquiza, A. La organización de las organizaciones sociales Aplicaciones desde perspectivas sistémicas. Santiago: Ril editores.

ARnold, M. (2000). Las universidades como sistemas sociales: Estructura y semántica. Revista Mad. No 2. Universidad de Chile, Departamento de Antropología.

Bauman, Z. (1999). La globalización consecuencias humanas. Buenos Aires: FCE.

Banco Mundial (2017). Estrategia de educación 2020 del Banco Mundial. Versión preliminar del resumen. Recuperado de http://siteresources.worldbank.org/ EDUCATION/

Banco Mundial (1999). El desarrollo al servicio del conocimiento. BM: Washington D.C. Recuperado de http://documentos.bancomundial.org/curated/es/

Banco Mundial (1995). La enseñanza superior: Las lecciones derivadas de la experiencia. BM: Estados Unidos. Recuperado de http://documentos.bancomundial.org/ curated/es/ 
Barbano, G. (1999). La educación superior en la segunda mitad del siglo XX. Los alcances del cambio en América Latina y el Caribe. Revista Iberoamericana de Educación. No 21. OEI. Recuperado de https://rieoei.org/historico/documentos/ rie21a01.htm

BASADRE, J. (1975). La vida y la historia. Lima: Banco Internacional.

BЕск, U. (1998). ¿Qué es la globalización? Falacias del globalismo, respuestas a la globalización. Barcelona: Paidós.

Bialakowsky, A. (2014). "Crisis global y cambio civilizatorio. Teoría y praxis intelectual”. Revista de Sociología. Vol. XIX N 24. Lima: UNMSM FCS E.A.P. de Sociología.

Bourdieu, P. (1991). La distinción. Criterio y bases sociales del gusto. Madrid: Taurus Humanidades.

Bourdieu, P. y Passeron, J. (1967). Los estudiantes y la cultura. Barcelona: Editorial Labor.

Bourdieu, P. y Passeron, J. (1977). La reproducción. Elementos para una teoría del sistema de enseñanza. Barcelona: Laia.

Boron, A. (1997). La globalización, ¿fase superior del imperialismo? Chomsky, N. y Dieterich, H. (1997). La sociedad global. Buenos Aires: Líber/Arte CBC

Briceño, R. y Sonntag, H. (1999). El legado de la sociología, la promesa de la ciencia social. Caracas: Nueva Sociedad.

Brunner, J. (2000). Notas sobre la educación superior. Universidad de Antioquía: Medellín.

Castells, M. (2002). La galaxia Internet, empresalsociedad. Barcelona: Plaza\&Janés.

Castells, M. (2002). Cultura y Sociedad del conocimiento: presente y perspectivas de futuro. Debates Culturales, UOC. Recuperado de http://www.uoc.edu/culturaxxi/ esp/articles/castells0502/castells0502.html

Сномsкy, N. y Dieterich, H. (1997). La sociedad global. Buenos Aires: Liber/Arte CBC.

De Azevedo, F. (1997). Sociología de la educación. México: FCE.

Delors, J. (1996). La educación encierra un tesoro. Santillana, Ediciones UNESCO. Recuperado de http://unesdoc.unesco.org/images/0010/001095/109590so.pdf

De Rivero, O. (1998). Mito del desarrollo. Los paises invisibles en el siglo XXI. Lima: Mosca Azul Editores.

De Sousa Santos, B. (2007). La universidad en el siglo XXI. Para una reforma democrática y emancipatoria de la universidad. Bolivia: CIDES-UMSA. Recuperado de http://www.boaventuradesousasantos.pt/media/universidad_siglo_xxi-.pdf

Dieterich, Heinz (1997). Globalización, educación y democracia en América Latina. Сномsкy y Dieterich. Recuperado de https://books.google.com.pe/books/ about/La_aldea_global.html? 
Dubet, F. y Martuccelli, D. (1998). En la escuela. Sociología de la experiencia escolar. Buenos Aires: Losada.

Familiar, J. (2017). Momento decisivo: La educación superior en América Latina $y$ el Caribe. Recuperado de http://www.bancomundial.org/es/news/ press-release/2017/05/17/

Ferreyra, M.; Avitabile, C.; Botero, J., Haimovich, F. y Urzúa, S. (2017). Momento decisivo La educación superior en América Latina y el Caribe. Grupo Banco Mundial. Recuperado de https://openknowledge.worldbank.org/bitstream/handle/

Galeano, E. (1996). La teoría del fin de la historia se pone de moda. Critica de la modernidad y globalización. Lima: Ediciones del Salmón.

García, N. (1999). Globalizarnos o defender la identidad. Aproximaciones a la globalización. N 163. Venezuela: Nueva Sociedad.

Hirschman, A. (1991). Retóricas de la intransigencia. México: FCE.

Lerner, S. (2014). La universidad del siglo XXI. Recuperado de http://vicerrectorado. pucp.edu.pe/academico/noticias/aula-magna-2014

Lummann, N. (1998). Complejidady modernidad de la unidad a la diferencia. Valladolid: Editorial Trotta.

Mariátegui, J. (1973). 7 ensayos de la realidad peruana. Lima: Biblioteca Amauta.

Mariátegui, J. (1973). Ideología y politica. Lima: Biblioteca Amauta.

Morin, E. (2001). Los siete saberes necesarios a la educación del futuro. Bogotá: UNESCO.

Ortiz, R. (1996). Otro territorio, ensayos sobre el mundo contemporáneo. Buenos Aires: Universidad Nacional de Quilmes.

PÁsara, L. (2004). Luces y sombras de la cooperación internacional con nuestros países. Perú 21, Domingo 10 de octubre, Lima.

Petras, J. (2002). 11-S: Un año de construcción del imperio. Recuperado de www.rcci. net/ globalizacion/2002/ fg267.htm.

Picardo, O. (2000). Pedagogía informacional: enseñar a aprender en la sociedad del conocimiento. Recuperado de http://www.oei.es/historico/salactsi/opicardo2.htm

Quijano, A. (2014). Colonialidad del poder y clasificación social. Cuestiones y horizontes: de la dependencia histórico-estructural a la colonialidad/descolonialidad del poder. Buenos Aires: CLACSO. Editorial/Editor.

Quijano, A. (2000). Colonialidad del poder, eurocentrismo y América Latina. En Lander, L. Comp. La colonialidad del saber: eurocentrismo y ciencias sociales. Perspectivas Latinoamericanas. Buenos Aires: CLACSO.

Ríos, J. (1998). Actores y estructuras sociales en la globalización. Revista de Sociología, Vol. X. Lima: UNMSM.

Rojas, J. (2012). Sociedad bloqueada. Movimiento estudiantil, desigualdad y despertar de la sociedad chilena. Chile: Universidad de Concepción-RIL Editores.

Sen, A. (2000). Desarrollo y libertad. Barcelona: Planeta. 
Rodríguez, G. (2000). La educación superior en el siglo XXI. Líneas estratégicas de desarrollo. Una propuesta de la ANUIES. Revista de la Educación Superior. Vol. XXIX, No 113. Recuperado de https://www.google.com.pe/search?q=Rodríguez\% $2 \mathrm{C}+\mathrm{G} .+(2000)$.

SAAVEDRA, J. (2014). La nueva ley universitaria aprobada por el Congreso. ¿Qué trae la ley universitaria? Lima: Diario El Comercio, Domingo 5 de mayo.

Touraine, A. (1998). ¿Qué es el desarrollo? Lima: PUCP.

Touraine, A. (1997). ¿Podremos vivir juntos? La discusión pendiente: El destino del hombre en la aldea global. Buenos Aires: FCE.

Trahtemberg, L. (2014). La nueva ley universitaria aprobada por el Congreso. "No basta parchar el pasado". Lima: Diario El Comercio, Domingo 5 de mayo.

TünNermann, C. (1996). La educación superior en el umbral del siglo XXI. CRESALC/ Unesco. Caracas: Venezuela.

Unesco (2014). América Latina y el Caribe. Revisión Regional 2015 de la Educación Para Todos. Recuperado de http://unesdoc.unesco.org/images/0023/002327/232701s. pdf

Unesco (1998). El nuevo rumbo de las universidades. Fuentes. Recuperado en http:// unesdoc.unesco.org/images/0011/001136/113669S.pdf

Unesco (1998). La educación superior en el siglo XXI, Conferencia Mundial sobre Educación Superior. 5-9 octubre. París: Recuperado de http://unesdoc.unesco.org/ images/0011/001163/116345s.pdf

UnESCo (1998). La educación superior y el desarrollo humano sostenible. París: Recuperado de http://www.unesco.org/education/educprog/wche/principal/shd-s.html

Unesco (1997). Nuestra diversidad creativa. Informe de la Comisión Mundial de Cultura y desarrollo. Madrid: Ediciones Santa María.

UNESCO (1995). Documento de politica para el cambio y el desarrollo de la educación superior. Recuperado de http://unesdoc.unesco.org/images/0009/000989/098992s.pdf

VAlDÉs, B. (1996). Conocimiento es futuro. Hacia la sexta generación de los procesos de calidad. México: CCTC.

VAllejo, C. (1983). Obra poética completa. Lima: Mosca Azul Editores.

VILLASEÑOR, G. ((2003). Universidades latinoamericanas para una globalización alternativa. ALAS XXIV Congreso. Arequipa.

Wallerstein, I. (2012). La educación superior bajo ataque. La Jornada. Recuperado de http://www.jornada.unam.mx/2012/03/10/opinion/022a1mun

YARZÁBAL, L. (1999). Consenso para el cambio en la educación superior. Caracas: IESALC/ Unesco. 